\title{
Strong variable linear polarization in the cool active star II Peg
}

\author{
Lisa Rosén ${ }^{1}$, Oleg Kochukhov ${ }^{1}$ and Gregg A. Wade ${ }^{2}$ \\ ${ }^{1}$ Deptartment of Physics \& Astronomy, Uppsala University, \\ Box 516, SE-75120 Uppsala, Sweden \\ email: lisa.rosen@physics.uu.se \\ email: oleg.kochukhov@physics.uu.se \\ ${ }^{2}$ Department of Physics, Royal Military College of Canada, \\ PO Box 17000, Station Forces, Kingston, Ontario K7K 7B4, Canada \\ email: Gregg.Wade@rmc.ca
}

\begin{abstract}
Magnetic fields of cool active stars are currently studied polarimetrically using only circular polarization observations. This provides limited information about the magnetic field geometry since circular polarization is only sensitive to the line-of-sight component of the magnetic field. Reconstructions of the magnetic field topology will therefore not be completely trustworthy when only circular polarization is used. On the other hand, linear polarization is sensitive to the transverse component of the magnetic field. By including linear polarization in the reconstruction the quality of the reconstructed magnetic map is dramatically improved. For that reason, we wanted to identify cool stars for which linear polarization could be detected at a level sufficient for magnetic imaging. Four active RS CVn binaries, II Peg, HR 1099, IM Peg, and $\sigma$ Gem were observed with the ESPaDOnS spectropolarimeter at the Canada-France-Hawaii Telescope. Mean polarization profiles in all four Stokes parameters were derived using the multiline technique of least-squares deconvolution (LSD). Not only was linear polarization successfully detected in all four stars in at least one observation, but also, II Peg showed an extraordinarily strong linear polarization signature throughout all observations. This qualifies II Peg as the first promising target for magnetic Doppler imaging in all four Stokes parameters and, at the same time, suggests that other such targets can possibly be identified.
\end{abstract}

Keywords. stars: magnetic field - stars: late-type - stars: individual: II Peg, HR 1099, IM Peg, $\sigma$ Gem - polarization

\section{Introduction}

Stellar magnetic fields can be detected by the appearance of signatures in the polarization spectra. The degree of polarization is proportional to the magnetic field strength and a weak field will hence result in weak polarization signatures. Cool stars generally have relatively weak magnetic fields which sometimes makes it difficult to detect any polarization. Since linear polarization is up to 10 times weaker than circular polarization, it is even more difficult to detect linear polarization. Because of this, the current studies of cool star magnetic fields are performed using circular polarization only.

This is not optimal since circular polarization is only sensitive to the line of sight component of the magnetic field vector. The information that can be extracted from circular polarization alone is hence not sufficient to provide a complete picture of the magnetic field geometry. The lack of information becomes especially problematic when the fields are complex which often is the case for cool stars. One of the major problems is cross talk, especially between radial and meridional field components, see e.g. Donati \& Brown (1997). 
The solution to the problem would be to include linear polarization since linear polarization is sensitive to the transverse component of the magnetic field vector. This means that additional information about the magnetic field geometry can be extracted and when combined with circular polarization the picture of the magnetic field topology will be a lot more complete. This has been shown in several numerical experiments, see e.g. Kochukhov \& Piskunov (2002) and Rosén \& Kochukhov (2012). The inclusion of linear polarization will also remove the crosstalk.

In a previous study by Kochukhov et al. (2011) they managed to detect linear polarization in two cool stars. This study was important since it proved that detection of linear polarization in cool stars is possible. However, their linear polarization observations did not have a sufficient $\mathrm{S} / \mathrm{N}$ ratio to be useful for magnetic imaging. The goal of our study was therefore to take this one step further by identifying cool stars for which linear polarization could be detected at a level sufficient for magnetic imaging.

\section{Observations}

All our spectropolarimetric observations were performed at the Canada-France-Hawaii Telescope (CFHT) with the spectropolarimeter ESPaDOnS. ESPaDOnS has a spectral resolution of about 65000 and a wavelength coverage of $370-1050 \mathrm{~nm}$ for a single exposure.

We focused our initial observations on four RS CVn stars, II Peg, HR 1099, IM Peg and $\sigma$ Gem as they are among the most magnetically active cool stars.

Full Stokes observations of all four stars were performed in periods during 2012 February to July. During this time, II Peg and IM Peg were observed 3 times each, HR 1099 was only observed once and $\sigma$ Gem was observed 4 times.

As expected, we could not detect any linear polarization signatures in individual spectral lines. We therefore applied the multi-line technique called least-squares deconvolution (LSD) Donati et al. (1997). Assuming all lines are self-similar, they can be combined into one mean profile where each line is assigned an individual weight depending on wavelength, magnetic sensitivity and central depth. This procedure will significantly increase the $\mathrm{S} / \mathrm{N}$ ratio. In Fig. 1 the set of observations with the highest detection probability for each star is shown.

\section{Results}

$\sigma$ Gem turned out to have the weakest linear polarization signatures out of the four stars. Despite this, Stokes $Q$ was securely detected in the observation shown in Fig. 1.

The polarization signatures of IM Peg in Fig. 1 look convincing and the detection probability is high. The other two observations of IM Peg were however not as convincing. It should also be mentioned that each of the three observations of IM Peg were obtained at different rotational phases, indicating a varying activity level across the stellar surface. A hint of a Stokes $I$ profile of the secondary can be seen to the blue side of the primary.

HR 1099 was one of the stars that Kochukhov et al. (2011) previously detected linear polarization in. In this study we managed to get a similar detection. It should be noted that previous observations were obtained with HARPSpol which has a spectral resolution of more than 100000 and that the spectral resolution of ESPaDOnS is significantly lower (65 000). In Fig. 1 the secondary is clearly visible in Stokes $I$, but also, a polarization signature of the secondary can be seen in Stokes $V$.

II Peg really stands out since the linear polarization signatures are enormous compared to any of the other stars. The amplitudes of the Stokes $Q U$ profiles shown in Fig. 1 are actually among the smallest compared to the other II Peg observations. Since II Peg 

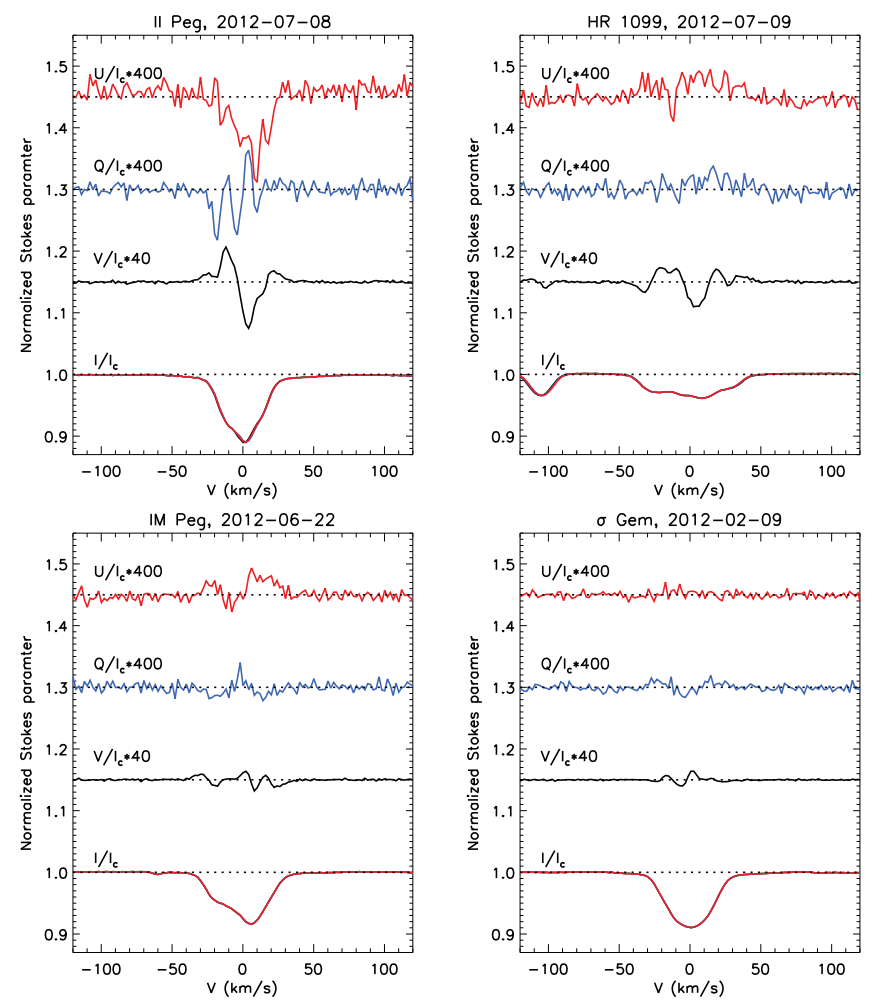

Figure 1. Representative sets of the LSD Stokes IQUV profiles for II Peg, HR 1099, IM Peg, and $\sigma \mathrm{Gem}$. The polarization profiles are magnified and shifted vertically.

proved to be a very promising target we decided to spend the remaining observations on this star.

\section{Further observations and analysis of II Peg}

We managed to get 7 more observations of II Peg in 2012 September and also an additional 2 in 2012 December/2013 January. The activity proved to be continuously high throughout all the observations. The mean amplitudes of Stokes $Q U$ were as large as the Stokes $V$ amplitudes for some of the other stars and the peak amplitude went as high as $8 \cdot 10^{-4} I_{\mathrm{c}}$. For a more detailed description of these observations, see Rosén et al. (2013).

Even though we had 12 complete sets of Stokes IQUV observations which all had a $\mathrm{S} / \mathrm{N}$ ratio sufficient for magnetic imaging, they were still not optimal for mapping. The reason is the long time period over which these observations were obtained, about 6 months. The field is constantly evolving and profiles obtained at the same rotational phase but at different observing epochs showed different shapes.

New observations were therefore obtained during 2013 June 15 to July 1. Once again the observations were done at CFHT with ESPaDOnS. During this period another 12 complete sets of observations were performed with good phase coverage. All observations can be seen in Fig. 2.

Once again the activity level was continuously high throughout the observing period. Clear distortions due to temperature inhomogeneities can be seen in all Stokes $I$ profiles. The Stokes $Q U V$ profiles vary in shape from one phase to the next. 

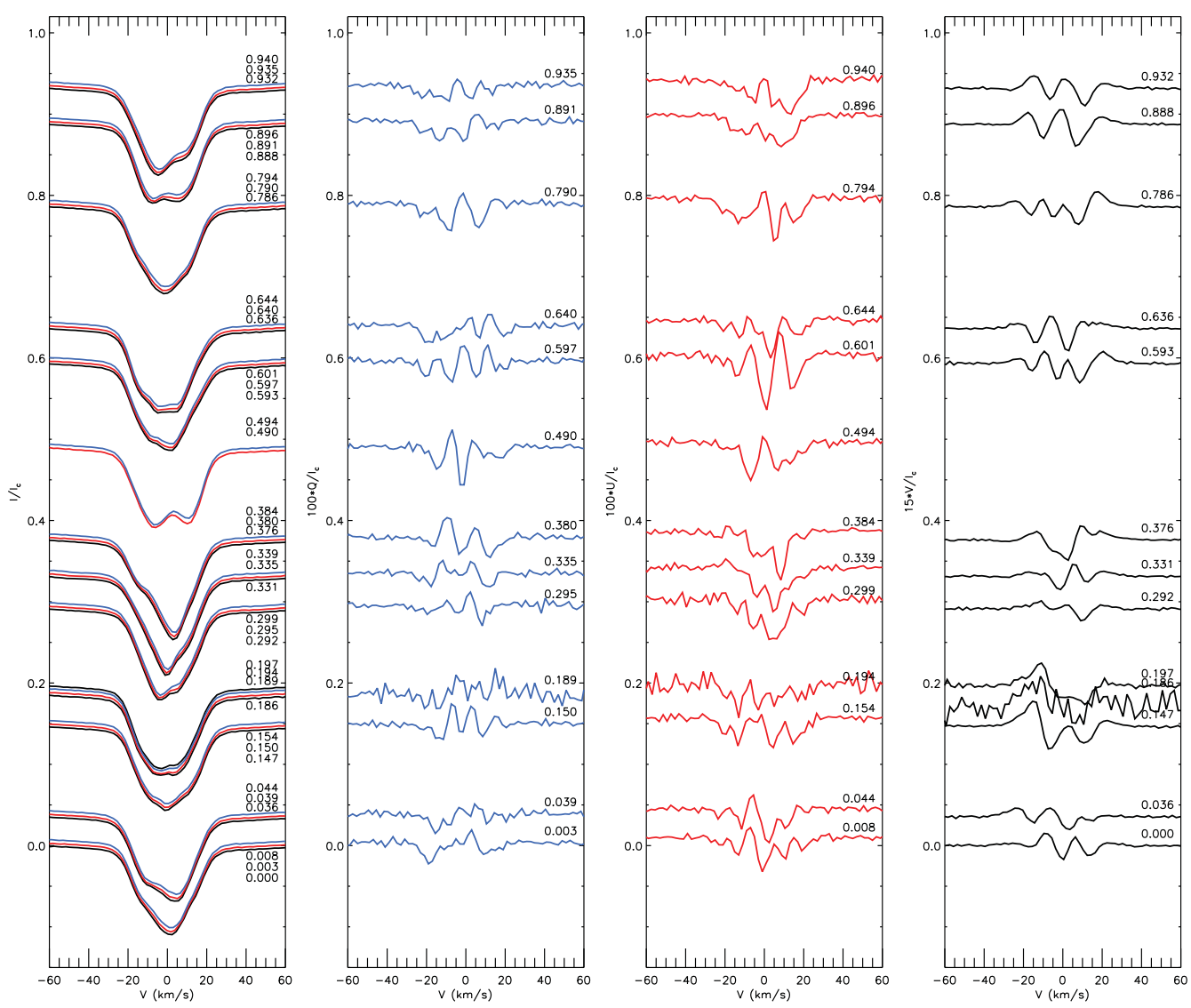

Figure 2. LSD Stokes $I Q U V$ profiles of II Peg. Each panel represents a different Stokes parameter. Profiles have been shifted vertically according to the rotational phase and the orbital radial velocity variation has been corrected for. Stokes $Q$ and $U$ (middle panels) have been magnified by a factor of 100 while Stokes $V$ (right panel) has been magnified by a factor of 15 .

\section{Conclusions}

This set of observations is unique since such a set has never previously been obtained for a cool star. It proves that linear polarization can be detected in cool stars at a level suitable for magnetic imaging. It also proves that such observations does not require an ultra-high spectral resolution, but instead can be done at a spectral resolution of about 65000 . The next step will be to reconstruct the magnetic field map using all 4 Stokes parameters self-consistently and simultaneously with temperature spots.

\section{References}

Donati, J.-F. \& Brown, S. F., 1997, A\& A, 326, 1135

Donati, J.-F., Semel, M., Carter, B. D., Rees, D. E., \& Collier Cameron, A., 1997, MNRAS, 291,658

Kochukhov, O. \& Piskunov, N., 2002, A\&\&A, 388, 868

Kochukhov, O., Makaganiuk, V., Piskunov, N., Snik, F., Jeffers, S. V., Johns-Krull, C. M., Keller, C. U., Rodenhuis, M., \& Valenti, J. A., 2011, ApJ, 732, L19

Rosén, L. \& Kochukhov, O., 2012, A\& A, 548, A8

Rosén, L., Kochukhov, O., \& Wade G. A., 2013, MNRAS 